

\title{
GNREL
}

\section{GeoRePORT Protocol Volume I: Background Document}

\author{
Katherine R. Young, ${ }^{1}$ Anna Wall, ${ }^{1}$ Aaron Levine, ${ }^{1}$ \\ Alex Badgett, ${ }^{1}$ and Patrick F. Dobson ${ }^{2}$ \\ 1 National Renewable Energy Laboratory \\ 2 Lawrence Berkeley National Laboratory
}

\section{Suggested Citation}

Young, Katherine R., Anna Wall, Aaron Levine, Alex Badgett, and Patrick F. Dobson. 2019. GeoRePORT Protocol Volume I: Background Document. Golden, CO: National Renewable Energy Laboratory. NREL/TP-4A00-72980.

https://www.nrel.gov/docs/fy19osti/72980.pdf.

NREL is a national laboratory of the U.S. Department of Energy Office of Energy Efficiency \& Renewable Energy Operated by the Alliance for Sustainable Energy, LLC

This report is available at no cost from the National Renewable Energy Laboratory (NREL) at www.nrel.gov/publications.

Contract No. DE-AC36-08GO28308
Technical Report NREL/TP-4A00-72980

September 2019

National Renewable Energy Laboratory 15013 Denver West Parkway Golden, CO 80401

303-275-3000 • www.nrel.gov 


\section{NOTICE}

This work was authored in part by the National Renewable Energy Laboratory, operated by Alliance for Sustainable Energy, LLC, for the U.S. Department of Energy (DOE) under Contract No. DE-AC36-08G028308. Funding provided by U.S. Department of Energy Office of Energy Efficiency and Renewable Energy Geothermal Technologies Office. The views expressed herein do not necessarily represent the views of the DOE or the U.S. Government.

This report is available at no cost from the National Renewable Energy Laboratory (NREL) at www.nrel.gov/publications.

U.S. Department of Energy (DOE) reports produced after 1991 and a growing number of pre-1991 documents are available free via www.OSTI.gov.

Cover Photos by Dennis Schroeder: (clockwise, left to right) NREL 51934, NREL 45897, NREL 42160, NREL 45891, NREL 48097, NREL 46526.

NREL prints on paper that contains recycled content. 


\section{ACKNOWLEDGMENTS}

This work was authored in part by the National Renewable Energy Laboratory (NREL), operated by Alliance for Sustainable Energy, LLC, for the U.S. Department of Energy (DOE) under Contract No. DEAC36-08G028308. Funding provided by the U.S. Department of Energy Office of Energy Efficiency and Renewable Energy Geothermal Technologies Office. The views expressed in the article do not necessarily represent the views of the DOE or the U.S. Government. This work was also supported by the U.S. Department of Energy Office of Energy Efficiency and Renewable Energy Geothermal Technologies Office under Contract DE-AC02-05CH11231 with Lawrence Berkeley National Laboratory (LBNL).

The authors wish to thank Colin Williams (U.S. Geological Survey); Paul Brophy (EGS, Inc.); Graeme Beardsmore (Hot Dry Rocks); Ann Robertson-Tait (GeothermEx); Ben Matek (Geothermal Energy Association); Mark Ziegenbein, Eric Hass, and Jay Nathwani (DOE); Brittany Segneri (New West Technologies); Juliet Newson (International Geothermal Association); Saboth Garg (Leidos Inc.); and numerous workshop participants for their insights and suggestions relating to this study. We also thank the NREL review team, including Amanda Kolker, Hilary Hatch Copeland, Emily Fenwick, Kendra Palmer, Scott Gossett, Emily Newes, Jeff Logan, and David Mooney.

The Geothermal Resource Portfolio Optimization and Reporting Technique (GeoRePORT) is a product of the considerable effort of many parties. Analysts at NREL, LBNL, and New West Technologies have been researching the information in this Protocol since 2012 and conducting meetings, outreach, reviews, and workshops, and analyzing and negotiating content to find solutions to divergent views.

The documents of the GeoRePORT Protocol reflect the intellectual contributions of these many players. These end products have been enabled because of a shared vision that this work can make a significant contribution to advancing geothermal deployment. 
LIST OF ACRONYMS

ARRA

DOE

EGS

GEA

GeoRePORT

GRC

GTO

IGA

LBNL

NREL

RD\&D
American Recovery and Reinvestment Act

U.S. Department of Energy

enhanced geothermal system

Geothermal Energy Association

Geothermal Resource Portfolio Optimization and Reporting Technique

Geothermal Resources Council

Geothermal Technologies Office

International Geothermal Association

Lawrence Berkeley National Laboratory

National Renewable Energy Laboratory

research, development, and deployment 


\section{TABLE OF CONTENTS}

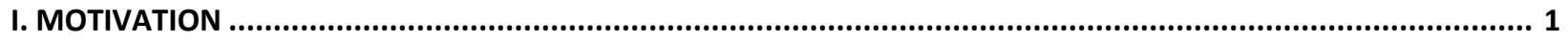

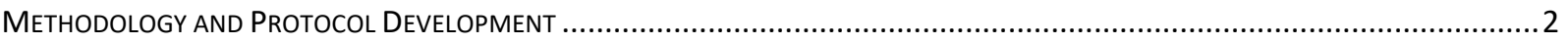

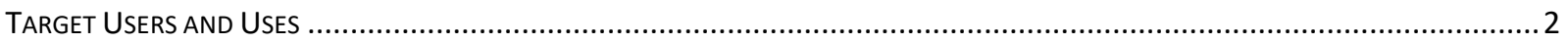

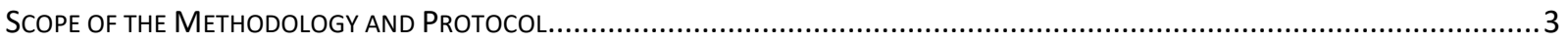

II. PRINCIPLES OF THE METHODOLOGY ......................................................................................... 4

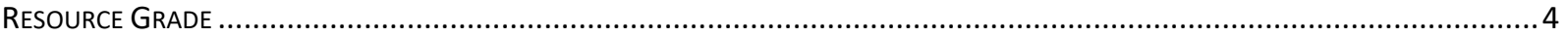

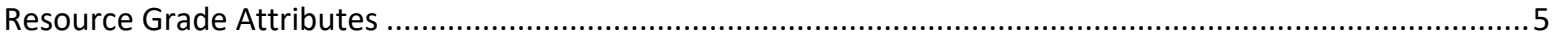

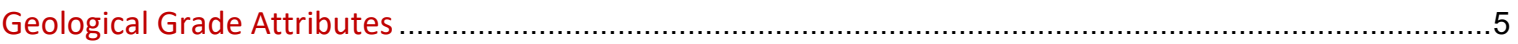

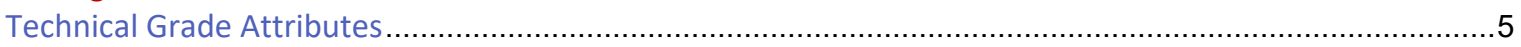

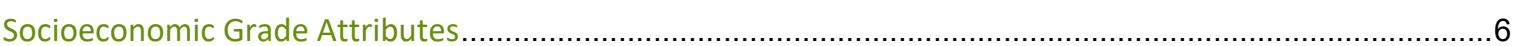

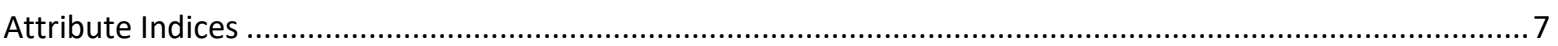

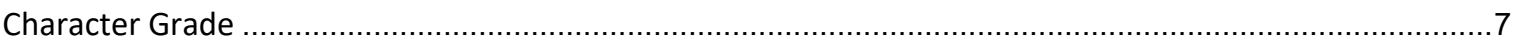

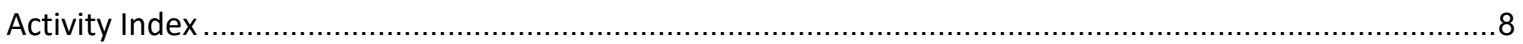

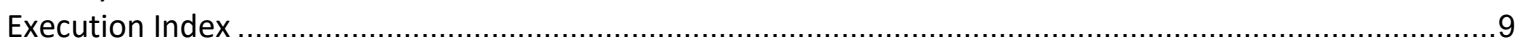

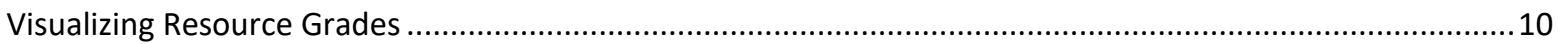

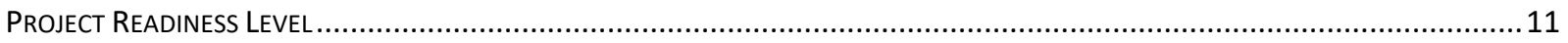

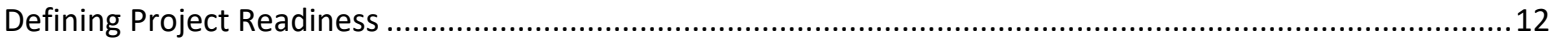

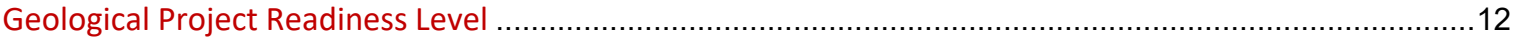

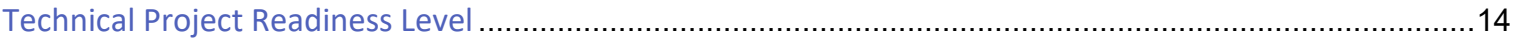

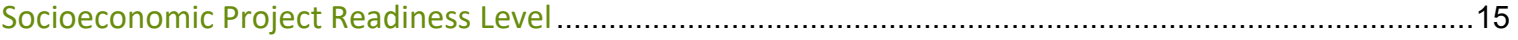

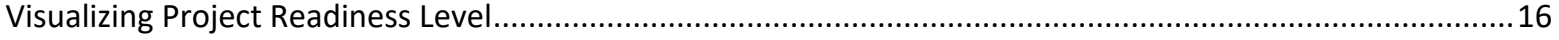

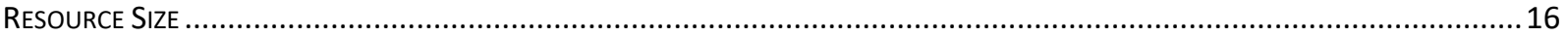

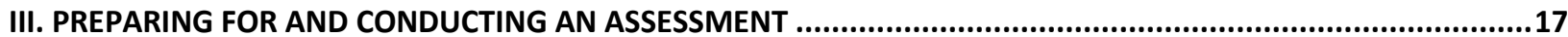

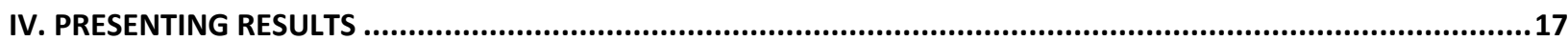

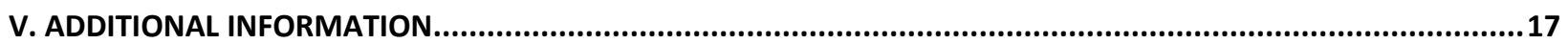

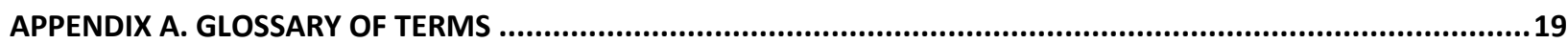

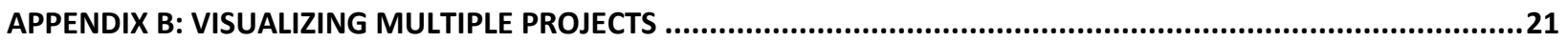

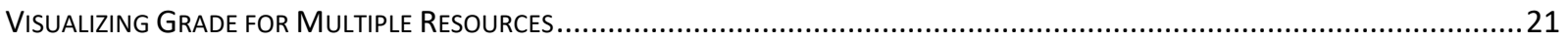

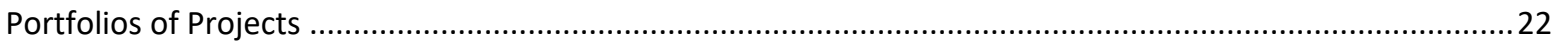




\section{LIST OF FIGURES}

FIGURE 1. EXAMPLE GRADE VISUALIZATION OF A HYPOTHETICAL RESOURCE USING A POLAR AREA CHART SHOWING THE FOUR GEOLOGICAL ATTRIBUTES.................................................................10

FIGURE 2. EXAMPLE OF A SUMMARY RESOURCE GRADE CHART. ......................................................11

FIGURE 3. PROJECT READINESS TRIANGLE DIAGRAM. ..................................................................16

FIGURE 4. EXAMPLE OF A RESOURCE GRADE DIAGRAM FOR MULTIPLE FICTITIOUS PROJECTS .........................21

\section{LIST OF TABLES}

TABLE 1. CHARACTER GRADES OF GEOLOGICAL ATTRIBUTES 7

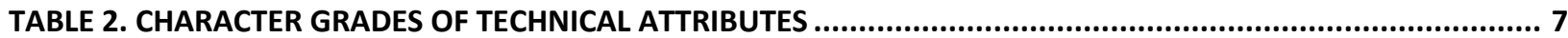

TABLE 3. CHARACTER GRADES OF SOCIOECONOMIC ATTRIBUTES .....................................................8

TABLE 4. EXAMPLE ACTIVITY INDICES OF THE TEMPERATURE ATTRIBUTE ...............................................8

TABLE 5. EXAMPLE EXECUTION INDICES FOR TEMPERATURE ACTIVITIES .................................................9.

TABLE 6. QUALIFYING CRITERIA TO MOVE BETWEEN LEVELS OF GEOLOGICAL PROJECT READINESS...................13

TABLE 7. QUALIFYING CRITERIA TO MOVE BETWEEN LEVELS OF TECHNICAL PROJECT READINESS .....................14

TABLE 8. QUALIFYING CRITERIA TO MOVE BETWEEN LEVELS OF SOCIOECONOMIC PROJECT READINESS .............15 


\section{MOTIVATION}

The U.S. Department of Energy's (DOE's) Geothermal Technologies Office (GTO) uses various metrics to track and measure impacts of its funding meant to catalyze innovation and private investment. As an example, one GTO goal is to "accelerate development of...undiscovered hydrothermal resources" (Williams et al. 2008b). For goals to be useful, however, it is important to be able to develop and measure baseline values, as well as incremental improvements, both at the individual project and aggregated portfolio levels. This need was the driving force behind the development of the Geothermal Resource Portfolio Optimization and Reporting Technique (GeoRePORT).

Though the Geothermal Energy Association (GEA) has developed Geothermal Reporting Terms and Definitions (GEA 2010) for reporting on project development, the U.S. geothermal industry has not adopted a systematic Protocol for resource reporting. Many countries, including Australia and Canada, have adopted geothermal resource reporting standards, and other natural resource industries (such as mining, oil, and gas) have standards and terminologies that are used to guide resource assessments. The United Nations Economic Commission for Europe has recently partnered with the International Geothermal Association to develop a Geothermal Specifications document for the United Nations Framework Classification system. All of these guides have provided valuable background information, insight, and influence in the development of the GeoRePORT Protocol.

The aim of the GeoRePORT Protocol is not to create a country-wide reporting standard for hydrothermal resources. Instead, it aims to best fit the needs of GTO in reporting impacts of its research, development, and deployment (RD\&D) activities.

The goals of the GeoRePORT Protocol documents are to:

- Provide a clear, objective, comprehensive, understandable (to technical and nontechnical audiences) Protocol for reporting geothermal resource grade and project readiness level

- Provide examples for using the methodology for GTO goal setting, measuring baselines, and reporting the impact of GTO-funded projects.

This Protocol will help GTO to:

- Quantitatively identify the greatest barriers to geothermal development

- Develop measurable program goals that will have the greatest impact to geothermal deployment

- Objectively evaluate proposals based (in part) on a project's ability to contribute to program goals

- Monitor project readiness level

- Report on GTO portfolio performance

- Communicate the impacts of funding decisions to nontechnical budget decision makers (e.g., U.S. Congress). 


\section{METHODOLOGY AND PROTOCOL DEVELOPMENT}

The idea of the development of this reporting methodology surfaced around 2010 after GTO received funding from the American Recovery and Reinvestment Act (ARRA 2009). Several ARRA projects were underway, and GTO needed a way to report the individual and cumulative impact of its funded research. Development of a reporting methodology, however, would take years and would need support and buyin from industry to be successful.

In 2013, GTO began more formal discussions with industry, hosting a resource reporting knowledge exchange and discussion in Sacramento, California, in conjunction with the Geothermal Resources Council (GRC) Resource Assessment and Optimization Workshop (June 2013), and discussions with the Boards of Directors of GEA and GRC at their joint meeting in Reno, Nevada (June 2013). Sufficient interest and support were provided to prompt funding of this effort by GTO in 2014.

In 2014, the National Renewable Energy Laboratory (NREL) partnered with Lawrence Berkeley National Laboratory (LBNL) and New West Technologies to begin developing this reporting methodology. These early versions of the GeoRePORT were circulated to select industry members (in the United States and internationally) and the GEA to solicit feedback on the direction of the methodology's development. Later drafts were presented during workshops at GEA (August 2014) and GRC events (September 2014).

After further refinement, a draft of the methodology was presented to and discussed at an international working group of the International Geothermal Association in Bonn, Germany (December 2014), and in Washington, D.C. (March 2015); during the 2015 World Geothermal Congress in Melbourne, Australia (Young et al. 2015); during a targeted workshop at the GTO Peer Review (May 2015); and through targeted discussions during the GEA Summit (June 2015). Continuous refinement has occurred along the way and has led to the development of a Protocol, which will consist of six documents. Four of the six documents have been released (Background, Geological Assessment Tool, Technical Assessment Tool, and Socioeconomic Assessment Tool), while the Resource Size Assessment Tool and Case Studies documents await funding.

We continue to test the Protocol and GeoRePORT tool on DOE's previous and current portfolio of RD\&D projects and refine it, as necessary, to overcome any issues identified in implementation of the Protocol. We welcome any feedback and suggestions from all stakeholders.

\section{TARGET USERS AND USES}

This reporting Protocol is written for use by the GTO. The methodology is intended to be sufficiently technical to be of use to industry professionals, and to the GTO in developing metrics, setting baseline values, evaluating funding applications, and in summarizing and reporting results. Therefore, the language in the Protocol documents is also intended to be understood by nontechnical audiences, including the U.S. Congress and the general public.

For individual GTO project managers, identifying resource grade and project readiness level at a given location is important to understanding the success of emerging technologies. This Protocol provides a means of evaluating projects based on three components of resource grade: geological, technical, and 
socioeconomic. This type of grading system, previously nonexistent in the geothermal industry, is critical to conveying the impacts of GTO research.

For GTO decision makers, identifying overall portfolio success is key to justifying ongoing RD\&D funding and to target future funding efforts. Differentiating geothermal resources by resource grade allows for selecting research areas directed at overcoming the largest barriers, impacting the greatest amount of resources. Objectively and quantitatively reporting project readiness level is therefore a useful way of conveying the impacts of GTO's efforts.

\section{SCOPE OF THE METHODOLOGY AND PROTOCOL}

The GeoRePORT provides a process for objectively appraising project readiness level and resource grade in individual geothermal areas. This methodology is:

- A method for assessing individual projects

- A set of assessment tools applicable to all stages of geothermal development

- A Protocol for evidence-based, objective assessment of a project's progress and grade.

The GeoRePORT is not:

- A standard, or a pass/fail mark-it only provides the means to identify characteristics of geothermal resources and associated projects

- A reporting requirement for anything beyond the needs of GTO-namely for baseline assessment, goal setting, application evaluation, project close-out summaries, and portfolio reporting

- A process or plan for geothermal exploration or development at any location

- A replacement for geothermal expertise in assessing project potential

- A set of new national or local regulatory reporting requirements.

The GeoRePORT Protocol consists of this Background Document and three assessment tools.

- Geological Assessment Tool

- Technical Assessment Tool

- Socioeconomic Assessment Tool 


\section{PRINCIPLES OF THE METHODOLOGY}

The Geothermal Resource Portfolio Optimization and Reporting Technique (GeoRePORT) system is based on the concept that a geothermal system can be described both in terms of the quality of the geothermal resource as it relates to the potential to extract heat (resource grade) and the progress of research and development over the lifetime of the project (project readiness level).

By assessing the major characteristics of a geothermal resource, categorizing the techniques used, and evaluating how well the research techniques were implemented, users can report a resource grade covering multiple geological, technological, and socioeconomic attributes that can be compared across play types and geothermal areas. The grade of each resource is intended to be refined, if needed, as new and better information is collected and interpreted.

By assessing the development activities of the project, users can report on past and planned incremental project readiness level. Like the resource grade, the project readiness level will continually be updated throughout the project lifetime.

Resource grade and project readiness level are reported for three assessment categories: geological, technical, and socioeconomic. Each category has specific criteria and guidelines for assessing both resource grade and project readiness level, as outlined in each of the following assessment tools (and associated colors):

- Geological Assessment Tool (representative color: red)

- Technical Assessment Tool (representative color: blue)

- Socioeconomic Assessment Tool (representative color: green).

These assessment tools are written for industry professionals assigned to report resource grade and project readiness level to GTO. The Protocol is meant to aid and provide consistency in the reporting process, and does not replace intelligent expertise in geothermal exploration, in project development, or in preparing and selecting data to report.

\section{RESOURCE GRADE}

Traditionally, the grade of a natural resource is defined by a combination of factors. For example, the grade of a mined ore is described as the ore's mineral concentration that can be technically recovered, and the grade of oil is described in terms of a combination of heavy to light and sweet to sour. We apply this multifactorial grading approach to geothermal resources by identifying "attributes" specific to each of the three assessment categories (geological, technical, and socioeconomic). 
The attributes used by the Protocol to describe a geothermal resource include the constraints on the quality of the geothermal resource, as well as the technical and socioeconomic characteristics that determine whether the heat in the system can be produced.

Each attribute is ranked on a scale of $A$ through $E$, with $A$ being the highest. An attribute grade of $A$ is not necessarily the "best" value for a specific project goal. Some business models or plant designs may target grades lower than A for some or all of the attributes. Examples include:

- $\quad$ Some developers may be interested in average temperature resources (Temperature Grade $=\mathrm{C}$ ) and poor fluid chemistry (Fluid Chemistry Grade $=\mathrm{D}-\mathrm{E}$ ) to take advantage of secondary mineral recovery potential from the geothermal brine.

- Near-field resources (resources located near operating plants) may have high temperatures (Temperature Grade $=\mathrm{A}$ ), but low permeability (Permeability Grade $=\mathrm{C}$ ) and may be candidates for the application of enhanced geothermal system (EGS) techniques.

- For some business models, a very high-temperature resource does not necessarily need to have a large volume to be economical; in fact, a small- or average-size, high-temperature resource could be a viable target.

As these examples indicate, each developer must evaluate which grades are appropriate for his/her target business model. Resources with all attribute grades equaling A rarely exist.

\section{Geological Grade Attributes}

The structural, geophysical, geochemical, and hydrological attributes of a geothermal resource are important in describing the Geological Grade. The following attributes represent the geologic constraints on the quality of the geothermal heat resource:

- Temperature: The in-situ reservoir temperature indicates the amount of energy carried by the geothermal fluid, and is thus a commonly used proxy for the available enthalpy of the fluid.

- Volume: The size of the reservoir (thickness and area/extent) is necessary for determining the quantity of heat available.

- Permeability: The permeability of the reservoir rock, often driven by the degree to which the formations are fracture-dominated, largely controls the accessibility and potential recovery of the heat.

- Fluid Chemistry: The geothermal brines and/or gases may be so corrosive or deposit such significant scales that specific tools or materials may have to be used, and special treatments may be necessary, increasing project costs.

\section{Technical Grade Attributes}

Attributes important to the feasibility of extracting the geothermal resource, such as depth to the reservoir, describe the Technical Grade. Even though some of these attributes are related to a system's geologic features, the influence of these items in developing geothermal resources may vary as technology improves and are therefore treated separately from a system's geological attributes Technical feasibility is related to the degree of challenges in producing the resource. It is also a function 
of the energy available in the system. The energy that can be extracted from the heat of a geothermal reservoir is related to the flow rate of fluid and the difference between the enthalpy of the fluid in the reservoir and at the surface (DiPippo 2004). Hence, the technical grade is a combination of attributes related to energy production technology and to technical challenges to development:

- Logistics: Consideration of physical barriers to reach a resource (e.g., weather, topography, elevation/slope, volcanic hazards), requiring advanced or specific tools or materials that potentially increase project costs.

- Reservoir Management: Production from a geothermal reservoir involves many management decisions such as makeup well drilling, well, stimulation, downhole pump capabilities, and injection schemes. These management decisions are all critical to determining how much heat may be extracted from the reservoir.

- Power Conversion: Specific generation technologies, such as for low temperature systems or EGS, as well as component technologies, such as air vs. water cooling, are all influential in determining whether the geothermal heat extracted can be efficiently and economically put to use for power generation.

- Drilling: The reservoir depth and rock properties strongly determine drilling and project development costs.

\section{Socioeconomic Grade Attributes}

Socioeconomic Grade attributes refer to a series of nontechnical aspects of geothermal development, such as land access, transmission availability, and local demand:

- Land Access: Leasing and real estate laws and policies, as well as local opposition, add additional complexity to beginning and/or continuing resource development.

- Permitting: Permitting requirements, certainty, complexity, and timelines can vary from location to location. Challenges in obtaining the necessary permits can create the potential for temporary or permanent delays in resource development.

- Transmission: Existing transmission lines may be far from potential geothermal areas, and even if they exist, they may require costly upgrades to take power generated from a new geothermal development.

- Market: The appetite for geothermal power is related to policy and incentives the price of electricity, and other factors which go into utility willingness to sign power purchase agreements. 
The GeoRePORT Protocol expands upon the concept of grade by considering not only the attributes listed above, but also how the attribute is measured and what is known about the quality of the data collected. We divide each attribute into three separate indices to address data confidence:

- Character Grade: Describes the character itself-i.e., what is the intrinsic measurement that best describes the geothermal reservoir?

- Activity Index: Qualitatively ranks activities used to assign the character index appropriate for each attribute-i.e., how well is the level of this attribute grade known?

- Execution Index: Compares the diligence with which the technique was executed for a given activity-i.e., how much do we know about the quality of execution of that technique?

Just as each attribute is assessed independently from other attributes for the purpose of the Protocol, these indices are also independently evaluated for each attribute. This Background Document gives an overview of the concepts of Character, Technique, and Execution Indices. For more detail on these indices, please see the Assessment Tool documents of this GeoRePORT Protocol.

\section{Character Grade}

For each attribute, the Character Grade uses quantitative and qualitative measurements describing the current project within the range of possible conditions encountered in geothermal resources and projects. For example, a resource with a high temperature measurement is given a temperature character grade of $A$, while a resource with a low temperature would be assigned a temperature character grade of $\mathrm{E}$. The tables below list the character indices of each attribute.

Table 1. Character Grades of Geological Attributes

\begin{tabular}{lllll}
\hline & Temperature & Volume & Permeability & Fluid Chemistry \\
\hline A & $>300^{\circ} \mathrm{C}$ or steam & $>10 \mathrm{~km}^{3}$ & Very high & Ideal \\
\hline B & $230-<300^{\circ} \mathrm{C}$ & $>5-10 \mathrm{~km}^{3}$ & High & Favorable \\
\hline C & $150-<230^{\circ} \mathrm{C}$ & $0.5-2.5-5 \mathrm{~km}^{3}$ & Medium & Challenging \\
\hline D & $90-<150^{\circ} \mathrm{C}$ & $2.5-5 \mathrm{~km}^{3}$ & Low & Difficult \\
\hline E & $<90^{\circ} \mathrm{C}$ & $\leq 2.5 \mathrm{~km}^{3}$ & Very low & Acidic/caustic \\
\hline
\end{tabular}

Table 2. Character Grades of Technical Attributes

\begin{tabular}{lllll}
\hline & Drilling & Logistics & $\begin{array}{l}\text { Reservoir } \\
\text { Management }\end{array}$ & Power Conversion \\
\hline $\mathrm{A}$ & $<2 \mathrm{~km}$ & Ideal & Ideal & $>18 \%$ \\
\hline $\mathrm{B}$ & $2-3 \mathrm{~km}$ & Favorable & Favorable & $16-18 \%$ \\
\hline $\mathrm{C}$ & $3-4 \mathrm{~km}$ & Challenging & Moderate & $14-16 \%$ \\
\hline $\mathrm{D}$ & $4-5 \mathrm{~km}$ & Difficult & Difficult & $11-14 \%$ \\
\hline $\mathrm{E}$ & $>5 \mathrm{~km}$ & Impossible & Very difficult & $<11 \%$ \\
\hline
\end{tabular}


Table 3. Character Grades of Socioeconomic Attributes

\begin{tabular}{ll|l|l|l}
\hline & Land Access & Permitting & Transmission & Market Conditions \\
\hline A & Ideal & $\begin{array}{l}\text { No permitting barriers } \\
\text { present }\end{array}$ & Ideal & Favorable \\
\hline B & Favorable & $\begin{array}{l}\text { Manageable permitting } \\
\text { barriers }\end{array}$ & Favorable & Manageable \\
\hline C & Challenging & Permitting barriers present & Challenging & Acceptable \\
\hline D & Difficult & Difficult permitting barriers & Difficult & Difficult \\
\hline E & Very difficult & $\begin{array}{l}\text { Extreme permitting } \\
\text { barriers }\end{array}$ & Very difficult & Very difficult \\
\hline
\end{tabular}

\section{Activity Index}

The Activity Index describes the common activities used to understand the character attributes-both directly (measured values) and indirectly (by proxy). For example, exploration methods to evaluate temperature include remote sensing, surface hydrothermal manifestations surveys, geothermometry, and downhole temperature measurements. This Protocol lists the techniques and their associated index values, ranked by the likelihood that the techniques represent the actual attribute being described.

We expect that exploration programs will use more than one technique for estimating an attribute's character, and therefore the criteria developed accommodate such combinations. Table 4 provides an example of activity indices for the temperature attribute. Details of the activity indices for all attributes are provided within the related Assessment Tools of this Protocol; for example, the Geological Assessment Tool describes the activity indices for the temperature, permeability, volume, and fluid chemistry attributes.

Table 4. Example Activity Indices of the Temperature Attribute

Temperature Technique Indices

A Measured temperatures: Downhole temperature probe readings (well[s] drilled into reservoir)

B Estimated temperatures: Geothermometry (geothermal brines and gases)

C Estimated temperatures: Geothermometry (immature or mixed fluids, inconsistent results between geothermometers)

D Extrapolated temperature: Thermal gradient holes/well(s), alteration mineral assemblages, stable isotopes, fluid inclusion data

E Regional heat flow data 


\section{Execution Index}

The Execution Index describes how well an activity was implemented. Potential errors and uncertainties in data collection may be associated with certain information sources. For each technique used to measure an attribute, the execution indices reflect an understanding of how much is known about the data and provide a baseline understanding of how the data were verified in relation to the best practices associated with a given technique.

For example, results of sulfate-water oxygen isotope geothermometry could show little variation, resulting in temperatures with few outliers and suggesting high confidence in the results. However, these could be impacted by shifts in the oxygen isotope compositions of the water and/or the sulfate caused by processes such as boiling, dilution, and bacterial activity, which would render these results less reliable. In this case, a moderate value of the execution index could be assigned to represent this mixed confidence in the geothermometry conclusions. Table 5 provides an example of execution indices for one activity (subsurface temperature probe readings) used to estimate temperature.

Table 5. Example Execution Indices for Temperature Activities

Execution Index: Downhole Temperature Probe

A Probe allowed to equilibrate

- Cuttings and/or geophysics confirm measurement within the reservoir (i.e., downhole alteration mineralogy consistent with reading)

- Repeated surveys at the same well/location

- Frequent calibrations completed that follow a prescribed set of procedures.

B Probe allowed to equilibrate

- Cuttings and/or geophysics have not confirmed measurement within the reservoir (i.e., downhole alteration mineralogy not consistent with readings)

- Singular survey at the well/location

- Frequent calibrations completed, but prescribed set of procedures are not consistently followed.

C - Results taken from previous third-party studies of the area (either literature or contractors) with little or limited information on survey methods, replication, or error.

D Probe not allowed to equilibrate

- Cuttings and/or geophysics have not confirmed measurement within the reservoir

- Calibrations are not completed regularly, and no prescribed set of procedures exist.

E Assumed from studies of analogous geothermal settings or extrapolated from studies of nearby areas. 


\section{VISUALIZING RESOURCE GRADES}

Resource grades can be visualized using a polar area chart (Figure 1), in which each quadrant represents one of the four attribute grades for a category and is subdivided to show the values for the character, activity, and execution indices.

The diagram allows for quick assessment of the strengths and weaknesses of an area by scanning the darkly shaded wedges. In Figure 1, the temperature and volume resource grades of the reservoir are high, the permeability is about average, and the fluid quality/quantity is low.

By reviewing the lightly shaded areas, one can get a glimpse of the certainty of these values and understand where additional work may be needed to better understand the geothermal system. For example, the lightly shaded areas in Figure 1 show uncertainty in volume and permeability.

In the context of GTO's need for metrics, the polar area chart can be used to understand RD\&D impacts at a particular location, showing how the information for a given area has changed in response to the results of funded projects. For example, GTO could be able to clearly identify whether changes in the reported temperature are due to better measurements from new techniques. The chart allows users to quickly identify increases in information from one period to the next and may illustrate differences between seemingly similar projects (e.g., two projects with the same character indices may have vastly different activity and execution indices).

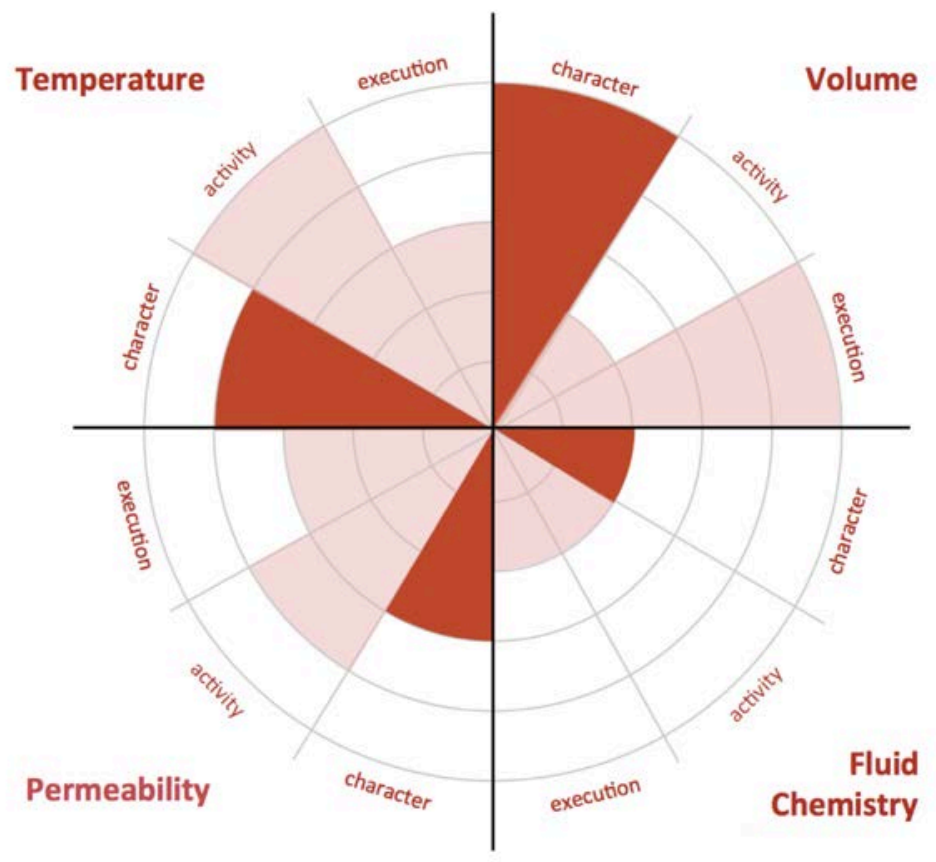

Figure 1. Example grade visualization of a hypothetical resource using a polar area chart showing the four geological attributes. Each quadrant represents a different geological attribute and is subdivided to show the character, activity, and execution index values. The darkly shaded wedges indicate the grade of the four resource attributes, while the lightly shaded

wedges indicate certainty (activity and execution). $E$ is located at the center of the circle, and $A$ is located along the circumference of the circle-the larger the shaded area, the better the resource. 
Each of the three categories (geological, technical, socioeconomic) will have its own similar polar area chart showing the respective character grade and certainty wedges. Detailed polar area charts for each category are provided in the Protocol documents.

All of the character attributes can be shown on a single summary chart (Figure 2). This summary chart shows only the character grades - not the activity or execution indices-so no uncertainty is illustrated in this figure.

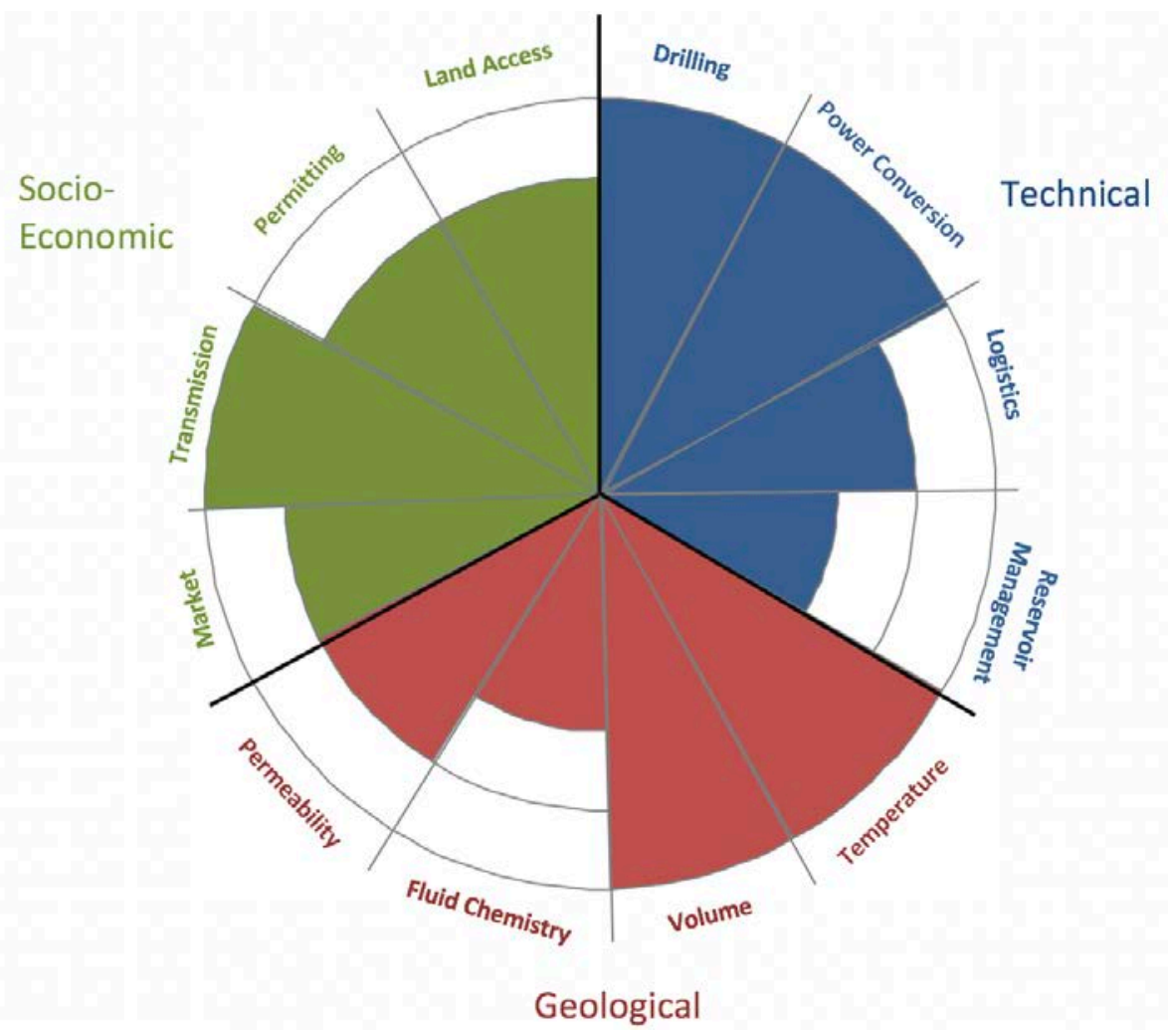

Figure 2. Example of a summary resource grade chart. The character grades for each of the twelve resource attributes are displayed in a single polar area chart. $E$ is located at the center of the circle, and $A$ is located along the circumference of the circle-the larger the shaded area, the better the resource. Since activity indices and execution indices are excluded from the diagram, no uncertainty is depicted.

\section{PROJECT READINESS LEVEL}

Like the geothermal grade, the GeoRePORT breaks the concept of project readiness into three assessments: geological, technical, and socioeconomic. As projects progress from one phase to the next, they pass through "activity thresholds" - minimum activities required to qualify for the next project readiness level category. For example, the difference between an undiscovered resource and inferred resource is the completion of some form of field sampling. 


\section{DEFINING PROJECT READINESS}

Project Readiness is defined for each of the three assessment categories: geological, technical, and socioeconomic. For each category, numerous qualifying criteria are defined in order to represent six different levels (0-5) of project readiness: unassessed, undiscovered, inferred, measured, tested, and examined. This section describes only the major concepts of the project readiness levels. Further details on their application to projects can be found in the related Geological, Technical, and Socioeconomic Assessment Tools.

\section{Geological Project Readiness Level}

The Geological Project Readiness Level's qualifying criteria describe exploration activities at a project location. The qualifying criteria are used to indicate the amount of activity that has occurred in an area and not whether those activities found a geothermal resource. Geological readiness levels are rarely downgraded because, for example, the difference between an undiscovered resource and an inferred resource is the completion of some form of field sampling; once sampling is completed, the project cannot return to the undiscovered category. The associated qualifying criteria for geological project readiness are shown below. 
Table 6. Qualifying Criteria to Move Between Levels of Geological Project Readiness

Geological Project

Readiness Level

G1 Undiscovered

\section{Qualifying Criteria}

For a resource to be considered "Undiscovered," the potential is estimated by at least one of the following activities:

1. Field mapping-structural features, hydrothermal alteration surface manifestations, etc.

2. Shallow heat flow studies (2-m probe)

3. Extrapolation of third-party data

4. Remote sensing.

Field Testing/Sampling

For a resource to be considered "Inferred," both of the following criteria must be met:

1. Resource temperature is estimated using at least one of the following methods:

G2

Inferred

a. A well-executed geothermometry study

b. Thermal gradient holes

2. Conceptual model of the system is supported by data from surface geophysical surveys.

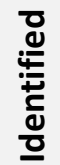

Slim/Core Hole into the Reservoir

For a resource to be considered "Measured," all of the following criteria must be met:

1. Temperature is measured at the reservoir level using the following methods:

a. Downhole probe in slimhole(s) drilled into the reservoir

G3

Measured

2. Temperature is corroborated using at least one of the following methods:

a. Geothermometry (preferably using well fluids

b. Assessment of hydrothermal mineral assemblages taken from cores and/or cuttings.

\section{Full-Diameter Well/Well Test}

For a resource to be considered "Tested," all of the following criteria must be met:

1. At least one full-diameter well has been drilled into the reservoir

G4 Tested

2. The reservoir permeability has been evaluated with at least one of the following methods:

a. Flow tests and/or

b. Pressure build up/draw down.

\section{Multiple Full-Diameter Wells Drilled}

G5

Examined

For a resource to be considered "Examined," the following criterion must be met:

1. Two or more full-scale wells must be drilled and flow tested. 


\section{Technical Project Readiness Level}

The Technical Project Readiness Level's qualifying criteria measure specific technical milestones in the development of a geothermal project. These technical milestones are chosen to mark technical progress for each project's phase (for example, testing of a well or a reservoir that produces sufficient flow for the project goals). Projects can also move backward in maturity if, for example, the reservoir no longer produces at the anticipated rate. For more detailed descriptions of each step, please see the Technical Assessment Tool.

Table 7. Qualifying Criteria to Move Between Levels of Technical Project Readiness

\begin{tabular}{|c|c|c|}
\hline \multicolumn{2}{|c|}{$\begin{array}{l}\text { Technical Project } \\
\text { Readiness Level }\end{array}$} & Qualifying Criteria \\
\hline \multirow[t]{2}{*}{ T1 } & $\begin{array}{l}\text { Unknown/ } \\
\text { unrecoverable }\end{array}$ & $\begin{array}{l}\text { Resource undeveloped. No drill holes, fluid chemistry, or flow tests have been } \\
\text { conducted to confirm existence/viability of the area. Geological assessment has been } \\
\text { performed, but no technical evaluation of the resource as an energy production site has } \\
\text { taken place. For a resource to be considered “Unknown/Unrecoverable," one of the } \\
\text { following criteria must be met: } \\
\text { 1. Site analysis completed including a geotechnical site analysis } \\
\text { 2. Site evaluated and determined not to have economic potential. }\end{array}$ \\
\hline & \multicolumn{2}{|c|}{ Promising Geophysical Surveys and Conceptual Model } \\
\hline T2 & Potential & $\begin{array}{l}\text { Surveying of the site confirms potential as an energy production site through } \\
\text { geophysical analysis. For a resource to be considered "Potential," the following criterion } \\
\text { must be met: } \\
\text { 1. Promising geophysical analyses and conceptual model completed. }\end{array}$ \\
\hline
\end{tabular}

\section{Successful Well Drilled into Reservoir}

For a resource to be considered "Discovered," the resource must meet initial

T3 Discovered temperature and permeability estimates. The following criterion must be met:

1. Well drilled into reservoir proves reservoir temperature and fluid flow.

\section{Well Field Drilled and Successfully Tested}

For a resource to be considered "Confirmed," all of the following criteria must be met:

T4 Confirmed 1. Two or more successfully drilled and tested wells

2. Production wells produce geofluids at necessary temperatures and flow rates for a minimum of 30 days.

Plant Development

For a resource to be considered "Demonstrated," power plant must be able to demonstrate integrated system operation. All of the following criteria must be met:

T5 Demonstrated

1. Well field and supporting infrastructure must be operational for a minimum of 30 days

2. Plant must produce power at or above initial power production estimates. 


\section{Socioeconomic Project Readiness Level}

The Socioeconomic Project Readiness Level's qualifying criteria identify specific political, economic, and social milestones in the development of a geothermal project. These socioeconomic milestones mark a level of maturity in planning, permitting, and agreements. Projects can also move backward along this scale if, for example, laws change or economic conditions evolve. For more detailed descriptions of each step, please see the Socioeconomic Assessment Tool.

Table 8. Qualifying Criteria to Move Between Levels of Socioeconomic Project Readiness
Socioeconomic
Project Readiness
Level

\section{Qualifying Criteria}
Resource undeveloped. For a resource to be considered "Unknown/uneconomic," one of the following criteria must be met:

\section{uneconomic}
S1 Unknown/
1. No site environmental (including a biological assessment and cultural resources study) or transmission interconnection analysis
2. Site evaluated and determined not to have economic potential (e.g., development unallowed, or having significant barriers).
Leasing and Transmission Analysis Complete
For a resource to be considered "Feasible," all of the following criteria must be met:
S2 Feasible
1. Environmental analysis required for leasing complete and the land is available for leasing or a lease is secured
2. Transmission interconnection analysis complete and determined to be economically feasible
3. Site evaluated and determined to have economic potential.

\section{Exploration and Drilling Permits Approved}

For a resource to be considered "Likely," the following criterion must be met:

\section{S3 Likely}

1. Permits approved for exploration (e.g., Notice of Intent) and well field drilling (e.g., Geothermal Drilling Permit), which includes associated environmental analysis.

\section{Well Field Drilled and Power Purchase Agreement Secured}

For a resource to be considered "Commercial," the following criteria must be met:

S4 Commercial

1. Approval of a Utilization Plan for construction and operation and a Commercial-Use Permit (if on a federally managed mineral estate), AND

2. Approval of any state- or local-level permits/approvals for construction, operation, and sale of the resource, OR

3. Power purchase agreement secured with off-taker.

Plant Development

For a resource to be considered "Secured," the resource must receive all necessary approvals from any federal and state authorities. The following criteria must be met:

S5 Secured

1. Approval of a Utilization Plan for construction and operation and a Commercial-Use Permit (if on a federally managed mineral estate)

2. Approval of any state- or local-level permits/approvals for construction, operation, and sale of the resource

3. Power purchase agreement secured with off-taker. 
Project readiness level can be visualized on a triangle diagram with geological progress shown on the $x$ axis, technical progress on the $y$-axis, and socioeconomic progress on the z-axis, as shown in Figure 3. Each project can be represented within a single triangle diagram, and dots can move independently along each of the three axes as a project progresses.

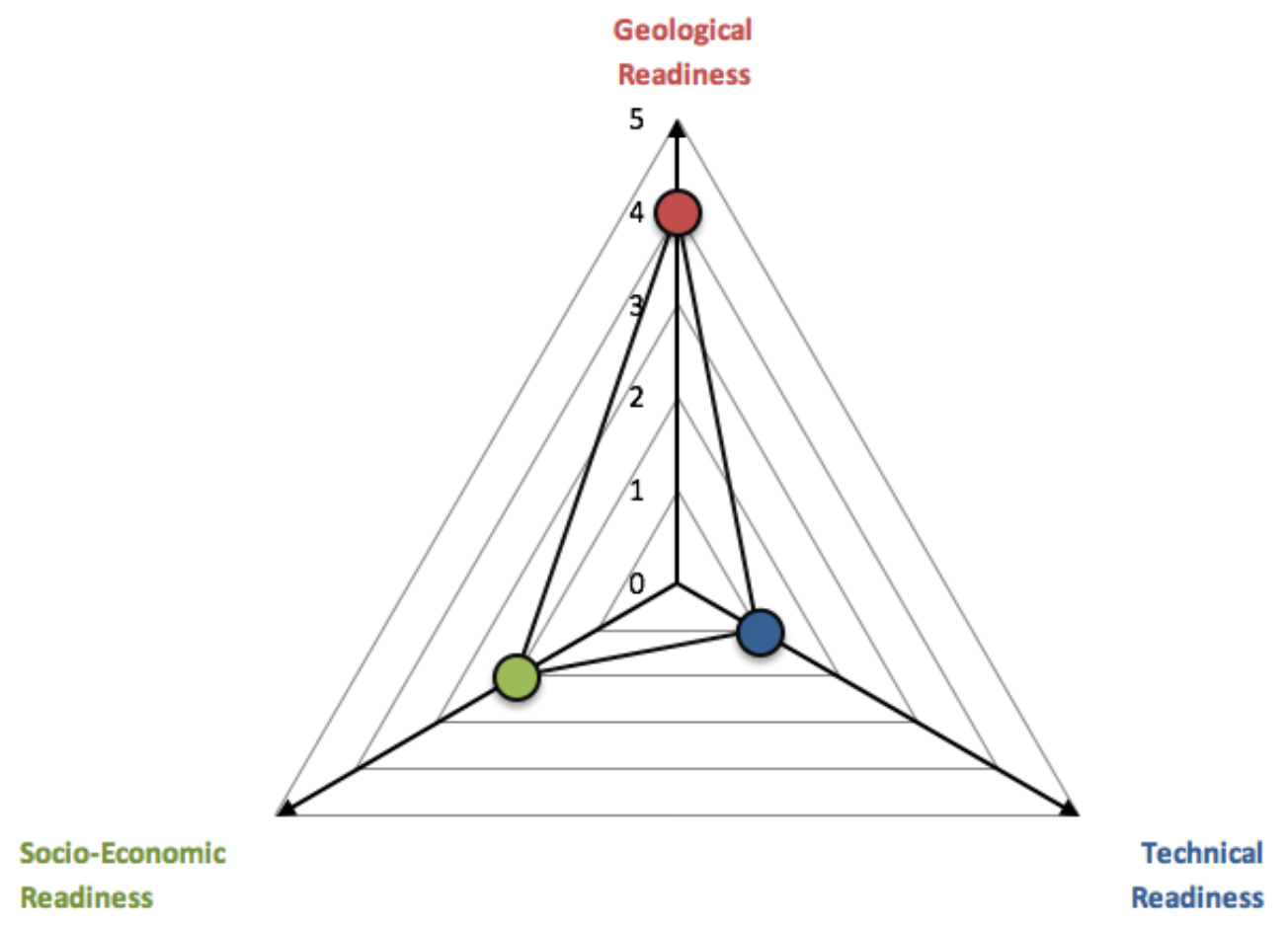

Figure 3. Project readiness triangle diagram. Each of the three categories is represented along an axis. Each project can be represented within a single triangle diagram, and dots can move independently along each of the three axes as a project progresses. This example demonstrates a strong (4) Geological Readiness Level, a low (1) Technical Readiness Level, and a moderate (2) Socioeconomic Readiness Level.

\section{RESOURCE SIZE}

The quantity of recoverable heat for a geothermal resource-the resource size-is often calculated in the early stages of project development using the United States Geological Survey heat-in-place volume method (Williams et al. 2008a). While its accuracy has been debated, suggestions have primarily focused on revisions of the assumptions, and not on fundamental changes to the concept (Garg and Combs 2015.) As more information is gathered at a geothermal location during the process of exploration, conceptual and numerical reservoir models are typically constructed, integrating multidimensional reservoir characteristics.

The Resource Size Assessment Tool of this Protocol is in development at the time of writing. This tool is expected to provide consistent resource size estimates to GTO across all projects. The Protocol is 
anticipated to allow for the reporting of resource size in several different units, including Joules, BTUs, $\mathrm{MWt}$, and MWe (assuming certain conversion efficiencies). This diversity will allow for the variety of potential geothermal end uses, from direct-use applications, such as space heating and drying applications, to electricity production.

\section{PREPARING FOR AND CONDUCTING AN ASSESSMENT}

Funding opportunity announcement applicants using this Protocol should be familiar with all of the Assessment Tools in this Protocol. As with all reporting Protocols, a qualified geothermal expert should be conducting the assessment.

More formal guidance on steps in preparing for and conducting an assessment will be developed in the future, based on review of application experience as well as better understanding of the needs and interests of the DOE Geothermal Technologies Office.

\section{PRESENTING RESULTS}

An Excel spreadsheet is under development at the time of writing to facilitate for reporting, visualizing, and presenting results outlined in this Protocol. The spreadsheet is to be populated using guidance in the Assessment Tools. The Assessment Tools guide the user in selecting inputs so that the results are repeatable by independent experts.

When completed, each resource assessment will consist of five pages:

1. Assessment Summary: Includes the summary grade chart and project readiness diagram

2. Geological Assessment: Includes geological progress and grade chart

3. Technical Assessment: Includes technical progress and grade chart

4. Socioeconomic Assessment: Includes socioeconomic progress and grade chart

5. Resource Size Assessment (under development): May include graphs of input (e.g., temperature, volume) and output values (e.g., quads, MWe).

\section{ADDITIONAL INFORMATION}

If you have questions, comments, or suggestions for improvement, please contact us. We plan to continue to test and update this Protocol. 


\section{REFERENCES}

DiPippo, Ronald. 2004. "Second Law assessment of binary plants generating power from lowtemperature geothermal fluids." Geothermics, v. 33, p. 565-586.

GEA. 2010. New Geothermal Terms and Definitions: A Guide to Reporting Resource Development Progress and Results to the Geothermal Energy Association. Geothermal Energy Association. http://geo-energy.org/pdf/NewGeothermalTermsandDefinitions January2011.pdf

ARRA. 2009. Pub. L. No. 111-5, 123 Stat. 115, 516 (Feb. 19, 2009).

Garg, S.K. and J. Combs. 2015. "A reformulation of United States Geological Survey volumetric "heat in place" resource estimation method." Geothermics, 55,150-158

Williams, C.F., M.J. Reed, and R.H. Mariner. 2008a. "A Review of Methods Applied by the U.S. Geological Survey in the Assessment of Identified Geothermal Resources." U.S. Geological Survey Open-File Report 2008-1296, 27 p.

Williams, C.F., M.J. Reed, R.H. Mariner, J. DeAngelo, and S.P. Galanis, Jr. 2008b. "Assessment of Moderate- and High-Temperature Geothermal Resources of the United States." U.S. Geological Survey Fact Sheet 2008-3082, 4 p.

Katherine Young, Anna Wall, Patrick Dobson, Mitchell Bennett, and Brittany Segneri. 2015. "Measuring Impact of U.S. DOE Geothermal Technologies Office Funding: Considerations for Development of a Geothermal Resource Reporting Metric." In: Proceedings, World Geothermal Congress. World Geothermal Congress; April 19, 2015; Melbourne, Australia. World Geothermal Congress. 
APPENDIX A. GLOSSARY OF TERMS

Activity Threshold:

Attribute:

Character:

Confirmed:

Demonstrated:

Discovered:

Execution:

Feasible:

Fluid Availability:

Geological:

Permeability:

Potential:

Project Readiness:
Minimum exploration activities required to qualify for the next mostadvanced project readiness level category.

A factor that represents geologic, technical, or economic constraints on the quality or feasibility of the geothermal heat resource.

The concept that each attribute of geothermal reservoir has an intrinsic measure or measurement that can embody or describe the in-situ geothermal reservoir.

Available observational geothermal data and evidence that "speak in favor of" or support scientific theories.

Geothermal resource qualities that are evident or established by arguments or reasoning; proven.

Observable patterns, correlations, or evidence as provided by geothermal resource data that lead to hypothesis formation and potential for scientific methodologies.

The concept that each technique used to measure a geothermal attribute brings a set of potential uncertainties and errors that may alter the representativeness of the results.

The likelihood of a geothermal resource, based on geological, technical, and socioeconomic grades, to be productive.

The accessibility and sustainability of fluid that can be used the transport heat from the reservoir.

Limiting factors associated with geologic constraints on the quality of the geothermal heat resource.

The degree to which reservoir formations control the accessibility and potential recovery of heat via fracture distribution and size.

A geothermal resource having or showing the capacity to be productive in the future.

A set of terminology that differentiates stages of exploration activities by their geologic, technologic, and socioeconomic features and that differentiates these stages by activity threshold. 
Resource Grade:

Resource Size:

Socioeconomic:

Technical:

Technique:

Temperature:

Volume:
The quality of the geothermal resource as it relates to the potential to extract heat, determined as a combination of resource attributes.

The quantity of recoverable heat for a geothermal resource.

Limiting factors that prevent or hinder further development and that are external to the resource itself or the technologies used to extract heat.

Limiting factors associated with current technology development that prevent or hinder access to producing a useable heat resource.

The scientific method and/or equipment used to measure a particular attribute.

In-situ temperature, enthalpy, and fluid phase of the reservoir, which indicates the quality of heat available for extraction.

Volumetric size of the constrained heat reservoir (via assessments of thickness and area), which indicates the quantity of available heat to be extracted. 


\section{APPENDIX B: VISUALIZING MULTIPLE PROJECTS}

Visualizing multiple projects-in terms of grade or progress-can be useful. While it may not be necessary for applying for funding, we include it in the Protocol in the event that it is needed.

\section{VISUALIZING GRADE FOR MULTIPLE RESOURCES}

For example, three projects can have the same resource size estimate, $50 \mathrm{MW}$, but have dramatically different geological attributes. In Figure 4, we represent the four geological attributes (temperature, volume, permeability, and fluid availability) for three different areas.

The first has a high temperature grade (A) and volume grade (A), but a low permeability grade (D). This may be the type of project that GTO targets for its EGS RD\&D portfolio.

The second resource is smaller, and has a volume grade of $B$, a permeability grade of $B$, and a temperature grade of $B$. This may be the type of resource that GTO targets for its hydrothermal RD\&D portfolio.

The last resource in this example is the lowest temperature resource, with a temperature grade of $C$, but is large (volume grade of A), and has a permeability grade of A. This may be the type of project that GTO targets for its low temperature RD\&D portfolio.

This graph allows users to quickly identify patterns in projects and may aid in "fingerprinting" sets of projects with similar characteristics to target for further research.

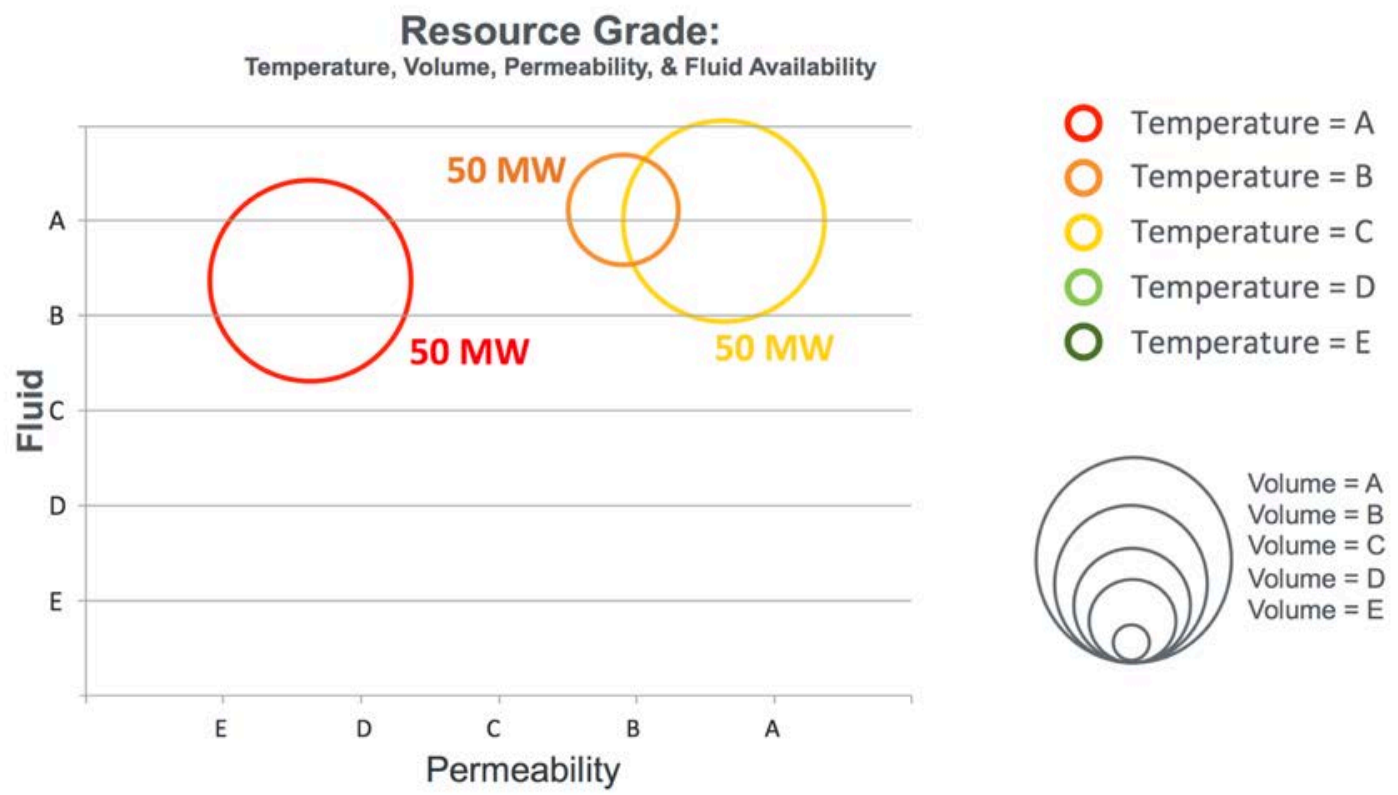

Figure 4. Example of a resource grade diagram for multiple fictitious projects 


\section{PORTFOLIOS OF PROJECTS}

The visualization displayed in Figure 4 is limited to a handful of projects; too many circles - for hundreds of geothermal areas-may make the graph busy and unreadable. For reporting on large data sets, for example the GTO portfolio of projects, the resource size estimates may be summed for particular grade attributes (e.g., all projects with a temperature grade of E) or sets of grade attributes (e.g., all projects with a temperature grade of $D$ or $E$, or all projects with a temperature grade of $A$ and permeability of $D$ ).

Similar, mean resource size estimates from multiple projects may be summed for a particular project readiness level point, for example all projects classified as "Inferred" or "Undiscovered."

It may be useful to sum mean resource size estimates for a particular combination of resource grade and project readiness level, for example projects with a temperature grade of E, classified as "Inferred."

It is also possible to combine resource grade and/or project readiness level with other geothermal classification schemes (e.g., play type) to help develop specific GTO metrics for increased geothermal deployment.

Using specific combinations of interest, GTO can:

1. Better understand the current potential for geothermal deployment

2. Set goals of overcoming certain project readiness level barriers and/or resource grade concerns

3. Evaluate project applications based on the project's proposed ability to overcome the barriers

4. Directly and objectively measure the impact of the RD\&D projects on overcoming these barriers

5. Quantitatively report on program achievements. 